\title{
NOVEL COMPOSITE MEMBRANES AND PROCESS FOR NATURAL GAS UPGRADING
}

\author{
Annual Progress Report-2002
}

\author{
Reporting Period: \\ $1 / 1 / 2002$ to $12 / 31 / 2002$ \\ Ben Bikson, Sal Giglia, and Jibin Hao \\ Issued: \\ March 2003
}

DOE Award Number:

DE-FC26-99FT40497

Submitting Organization:

Innovative Membrane Systems, Inc.

189 Dean Street, Norwood, MA 02062

and

UOP Gas Processing

25 East Algonquin Road, Des Plaines, IL 60017 


\section{DISCLAIMER}

This report was prepared as an account of work sponsored by an agency of the United States Government. Neither the United States Government nor any agency thereof, nor any of their employees, makes any warranty, express or implied, or assumes any legal liability for the accuracy, completeness, or usefulness of any information, apparatus, product, or process disclosed, or represents that its use would not infringe privately owned rights. Reference herein to any specific commercial product, process, or service by trade name, trademark, manufacturer, or otherwise does not necessarily constitute or imply its endorsement, recommendation, or favoring by the United States Government or any agency thereof. The views and opinions of authors expressed herein do not necessarily state or reflect those of the United States Government or any agency thereof. 


\section{ABSTRACT}

In the second phase of this project, the newly developed membrane module for natural gas dehydration was tested and evaluated in a pilot plant located at a commercial natural gas treatment site. This phase was undertaken jointly with UOP LLC, our commercialization partner.

The field test demonstrated that a commercial-size membrane module for natural gas dehydration was successfully manufactured. The membrane module operated reliably over 1000 psi differential pressure across the membrane in the field test. The effects of feed gas pressure, permeate gas pressure, feed flow rate, purge ratio (flow rate ratio of permeate outlet to feed), and feed gas dew point on the membrane module performance were determined and found to meet the design expectations. Although water vapor permeance was lower than expected, substantial natural gas dehydration was demonstrated with low purge ratio. For example, dew point was suppressed by as much as $30^{\circ} \mathrm{F}$ with only about 2 3\% purge ratio. However the bore side pressure drops were significantly higher than the projected value from the fluid dynamic calculation. It is likely that not all the fibers were open in either the sweep or the permeate tube sheet end. This could help to explain the relatively low water vapor permeances that were measured in the field.

An economic evaluation of the membrane process and the traditional Triethylene Glycol (TEG) process to dehydrate natural gas was performed and the economics of the two processes were compared. Two sets of membrane module performance properties were used in the economic analysis of the membrane process. One was from the results of this field test and the other from the results of the previous small-scale test with a medium pressure membrane variant conducted at $750 \mathrm{psig}$. The membrane process was competitive with the TEG process for the natural gas feed flow rate below 10 MMSCFD for the membrane with previously measured water vapor permeance. The membrane process was competitive for the feed flow rate below 1 MMSCFD even for the membrane with the water vapor permeance of this field test. 


\section{TABLE OF CONTENTS}

Disclaimer....................................................... 2

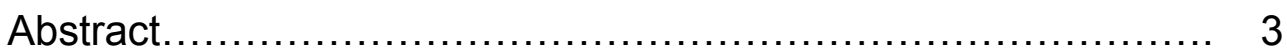

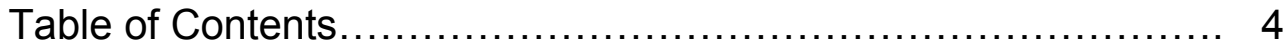

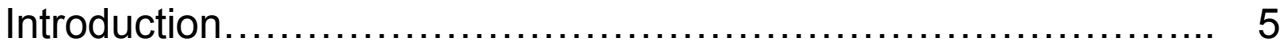

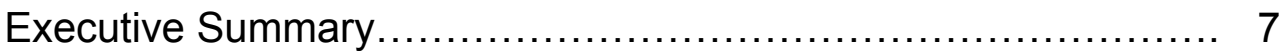

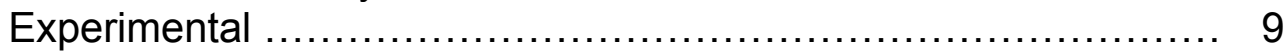

Field test plan for natural gas dehydration..................... 9

Field test site preparation.................................. 9

Pilot plant set-up and field test............................. 10

Results and discussions......................................... 11

Field test results and analysis............................... 11

Economic evaluation of natural gas dehydration............... 11

Conclusions........................................................ 15

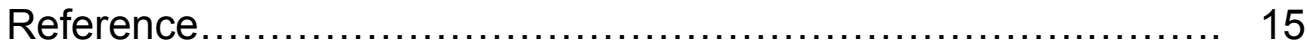

\section{PROPRIETARY APPENDICES}

Appendix A Test plan for natural gas dehydration

Appendix B Field test set-up

Appendix $C$ Field test and result analysis

Appendix $D$ Economic evaluation of natural gas dehydration 


\section{INTRODUCTION}

Innovative Membrane Systems, Inc. (IMS), a wholly owned subsidiary of Praxair, Inc., has developed novel technologies for the manufacture of two types of composite hollow fiber membranes that can be employed for natural gas sweetening and natural gas dehydration. One of these membranes is a composite polyimide hollow fiber developed for the removal of carbon dioxide from natural gas, i.e. for natural gas sweetening. The other membrane is a composite membrane with a unique hydrophilic separation layer specifically developed for natural gas dehydration. Both of these membranes are designed and constructed to operate in a high pressure natural gas environment. Because of their simplicity and modularity, membranes are highly suited to address current natural gas processing needs, especially for the small-size plant (below 10 MMSCFD) and for the plants located in a remote area.

Initially, a two-year $(\$ 650,000)$ research program was proposed to develop and demonstrate a novel membrane platform for natural gas treatment. The events of September 11 and other unexpected occurrences had a negative impact on the project schedule and the duration of the program was extended by 12 months, so that it now terminated on December 31, 2002. Phase I occupied the first 15 months of the program (2000 / 2001) and involved the development of high performance composite membranes for carbon dioxide removal from natural gas. The membrane development was further extended for natural gas dehydration. Phase II is the demonstration phase of the program, where the commercial potential of the new technology is demonstrated by the pilot scale field testing. This phase is undertaken jointly with our commercial partner, UOP LLC. The economic potential of the newly developed membrane technology for the treatment of natural gas is evaluated and compared to existing technologies.

At the end of the membrane development phase of the program, evaluation of the results prompted a refocus of program objectives. It was decided that the program benefits would be maximized if the focus of Phase II was to test a membrane system for natural gas dehydration in the pilot plant, rather than for carbon dioxide removal. This report covers the field demonstration of Phase II, test of membrane module performance at a commercial site for natural gas dehydration.

\section{Tasks for Year 2002: Phase II}

Task 1. Field test plan for natural gas dehydration

This task involves the development of a detailed field test plan. This test plan should list the required operating conditions, the required process variables for measurements, and procedure for testing the equilibrium and stability of the membrane module. The test plan should be initialed and developed by IMS, reviewed and accepted by UOP. 
Task 2. Pilot plant test preparation

This task includes design of a test site for natural gas dehydration, installation of on-line analytical equipment, and modification of pipeline and other hardware components. This is followed by detailed safety and engineering tests before the installation of the membrane module.

Task 3. Field test and data analysis

The field test should closely follow the test plan. The field test data should be digested and compared with the existing computer software of modeling natural gas dehydration. If necessary, the computer software should be revised to address the specific issues found at the test site. The computer software is then used to project the membrane module performance at operating conditions of interest.

Task 4. Economic evaluation of natural gas dehydration

This task involves the process design and economic evaluation of membrane natural gas dehydration. The economics of the membrane process was performed at a number of plant capacities and compared with the existing technology. 


\section{EXECUTIVE SUMMARY}

Innovative Membrane Systems, Inc., a wholly owned subsidiary of Praxair, Inc., has developed novel technologies for the manufacture of composite hollow fiber membranes that can be employed for the sweetening and dehydration of natural gas. In Phase I of this program, two composite polyimide hollow fiber membranes were developed for the removal of carbon dioxide from natural gas, i.e. for natural gas sweetening. A composite membrane with a unique hydrophilic separation layer specifically was also developed for natural gas dehydration. All the membranes are designed and constructed to operate in a natural gas environment.

Initially, a two-year $(\$ 650,000)$ research program was proposed to develop and demonstrate a novel membrane platform for natural gas treatment. The events of September 11 and other unexpected occurrences had a negative impact on the project schedule and the duration of the program was extended by 12 months, so that it now terminated on December 31, 2002. Phase I occupied the first 15 months of the program (2000 / 2001) and involved the development of high performance composite membranes for carbon dioxide removal from natural gas. The membrane development was further extended for natural gas dehydration. Phase II is the demonstration phase of the program, where the commercial potential of the new technology is demonstrated by pilot-scale field testing. This phase is undertaken jointly with our commercial partner, UOP LLC. The economic potential of the newly developed membrane technology for the treatment of natural gas is also evaluated and compared to existing technologies.

At the end of the membrane development phase of the program, evaluation of the results prompted a refocus of program objectives. It was decided that the program benefits would be maximized if the focus of Phase II was to field test a membrane system for natural gas dehydration in the field demonstration, rather than for carbon dioxide removal. This report covers the field demonstration of Phase II, testing the performance of membrane module at a commercial site for natural gas dehydration.

The field test demonstrated that a commercial-size membrane module for natural gas dehydration was manufactured successfully. The membrane module operated reliably over 1000 psi differential pressure across the membrane in the field test. The effects of feed gas pressure, permeate gas pressure, feed flow rate, purge ratio (flow rate ratio of permeate outlet to feed), and feed gas dew point on membrane module performance were determined and found to meet the design expectations. Although water vapor permeance was lower than the design expectation, substantial natural gas dehydration was demonstrated with a low purge ratio. For example, dew point was suppressed by as much as $30^{\circ} \mathrm{F}$ with only about $2 \sim 3 \%$ purge ratio. However the bore side pressure drop was much higher than the projected value from the flow dynamic calculation. It is likely that 
not all the fibers were open in either the sweep or the permeate tube sheet end. This could help to explain the relatively low water vapor permeance that was measured in the field.

An economic evaluation for the membrane process and the traditional TEG process to dehydrate natural gas was performed and the economics of the two processes were compared. Two sets of membrane module performance properties were used in the economic analysis of the membrane process. One was from the results of this field test and the other from the results of the previous small-scale test with a medium pressure membrane variant conducted at 750 psig. The membrane process was competitive for the feed flow rate below 10 MMSCFD for the membrane with previously measured water vapor permeance. The membrane process was competitive for the feed flow rate below 1 MMSCFD even for the membrane with the water vapor permeance of this field test. 


\section{EXPERIMENTAL}

The newly developed membrane module is tested and evaluated on a pilot scale for natural gas dehydration. The field test is conducted at a commercial natural gas treatment site. The procedures of the field test are described below.

\section{Field test plan for natural gas dehydration}

In order to evaluate the performance of the membrane module, a test plan was developed at IMS with the following objectives:

1) demonstrate mechanical and design integrity of the membrane module,

2) collect performance data in an actual natural gas stream for the purpose of:

a) comparing performance with process simulation models,

b) mapping system performance over a range of conditions to determine the effects of various operating parameters,

3) monitor long-term performance stability,

4) evaluate general operability of membrane module.

UOP reviewed the test plan and provided useful feedback on the test proposal. The field test plan was then finalized and implemented at a commercial natural gas treatment site.

\section{Field test site preparation}

UOP designed the pilot test system for natural gas dehydration testing and prepared the site for the field tests. The pilot test system formed part of a larger natural gas treatment facility. The testing procedure as well as the feed and product gas quality was integrated into requirements of the main facility. Thus, the dehydrated gas from the pilot test unit was post-treated to pipeline quality so that it could be returned to the main system. Similarly, the impact of potential failure of any part of the pilot test system on the main facility was carefully considered. Site preparation involved redesign of the test system, purchase and installation of analytical equipment, and completion of the necessary piping and other hardware changes.

UOP completed an engineering safety review of the test site and resolved the issues that had been identified. These issues included the control of feed pressure and permeate pressure, and humidification adjustment of the feed gas to the dehydration membrane module. The potential range of operating temperatures at the site was expected to vary during the course of the pilot testing. This necessitated giving special attention to the design of the natural gas feed system so that the required dew point of the feed and product streams could be maintained and accurately measured. Critical issues such as maintaining the desired degree of saturation of the feed gas, and minimizing the risk of condensing water vapor inside the membrane module were addressed. 


\section{$\underline{\text { Pilot plant set-up and field test }}$}

The field test skid was operated in parallel with the commercial membrane system. The natural gas feed stream of the test unit was introduced from the commercial plant feed stream. The feed stream of the test unit was first sent to the pretreatment unit to remove the entrained liquids (including heavy hydrocarbon liquids). The pretreated natural gas stream then was mixed with very fine water droplets that were generated from a water fog system (see following paragraph). The humidified natural gas was then introduced to a preheater so that the fine droplets completely evaporated and the natural gas feed stream reached the operating temperature required per the test plan. Humidity was measured after the pre-heater and adjustments to the water fog system were made until proper water vapor concentration in the natural gas feed was achieved. The pretreated, humidified warm feed gas then entered a single membrane pressure vessel that housed a single hollow fiber membrane module. As the natural gas flowed through the membrane module, water vapor was enriched in the lower-pressure permeate stream and collected in the permeate vent header. The permeate stream from the test unit was sent to the compressor to boost the pressure back to the feed pressure for further processing. The residual product from the test skid was the dehydrated natural gas that exited the membrane system with little pressure loss. The membrane module of the field demonstration was a four-port module. A fraction of the residual product was introduced to the permeate side as a sweep stream during the field test per the test plan.

The water fog generation system mentioned above included a metering pump and a fog nozzle. The metering pump was able to control water flow rate and pressurize water to 1500 2000 psi. The fog nozzle had the capacity to disperse the pressurized water into very fine droplets. In the field test, water was first pressurized in the metering pump and then fed to the fog nozzle. The dispersed water droplets from the fog nozzle were injected co-currently into the pretreated natural gas feed stream.

Prior to starting up the membrane unit, a high pressure leak test at about operating pressure was performed. Before startup with feed gas, the unit was purged with $\mathrm{N}_{2}$. In addition, the unit was properly grounded and purge gas $\left(\mathrm{N}_{2}\right)$ was being supplied to the electrical cabinet.

The pilot test was conducted according to the test plan developed for the membrane dehydration. During the test, when the test unit was operating at full feed pressure, the dehydrated natural gas product of the field test was treated and then combined with the product gas from the commercial unit. During tests at reduced feed pressure, the dehydrated natural gas was directed to the permeate by-product line for recompression and reprocessing. 


\section{RESULTS AND DISCUSSION}

\section{Field test results and analysis}

The membrane module for natural gas dehydration was first equilibrated for about one week at 750 psig and then tested at the baseline condition. No significant changes in membrane module properties were detected during the period of equilibration. The effects of purge ratio, feed flow rate, feed gas pressure, permeate gas pressure, temperature, and feed gas dew point on the membrane module dehydration performance were evaluated. Component permeances were calculated for each point. Most of the process variables tested did not have a significant effect on component permeances outside of the $\pm 10 \sim 20 \%$ scatter in the data. This confirmed that the model adequately accounted for most of the effects of the process variables.

The test results showed that the dew point suppression increased with decreasing feed flow, increasing purge ratio, and decreasing permeate pressure. Shell side pressure drop was measured to be essentially negligible at the base line conditions. This is consistent with the projected value from the fluid dynamic calculation.

While the design model (computer model) has properly predicted the relative effects of the various process variables, the absolute performance of the membrane module was lower than expected. The water vapor permeance was below the design expectation. Most likely this resulted from restriction of permeate and sweep flows. Not all fibers in either the sweep or permeate tube sheet end of the membrane module were sufficiently open to allow for unobstructed permeate and sweep flows. This was inferred from the permeate side pressure drop data, which was measured to be about double the projected value from the fluid dynamic model. If not all fiber bores were open then obviously not all the membrane area in the module was effectively utilized. However the analyses assumed the membrane module functioned properly when the water vapor permeance was calculated and thus the calculated permeance was much lower than the actual permeance. It should be noted that although the membrane module dehydration capacity was not as high as it potentially should have been, substantial dehydration at low purge ratio was demonstrated. For example, the dew point was suppressed by about $30^{\circ} \mathrm{F}$ with only about $2-3 \%$ purge ratio.

\section{Economic evaluation of natural gas dehydration}

The computer program employed to model the membrane natural gas dehydration process was validated by the field test data. This program was then used to model the membrane dehydration process at a number of operating conditions of interest. 
The membrane process considered in the economic evaluation of natural gas dehydration was very simple and consisted of three basic units: a pretreatment, a membrane module, and a permeate compressor. The natural gas feed was first pretreated before it was introduced into the membrane module. The retentate stream existing membrane module was the dehydrated natural gas. A fraction of the dehydrated natural gas (product) was used as a sweep gas in the dehydration process. This is because a minimum sweep volume is required so that the product (retentate) stream meets the pipeline specification for the water vapor content. In some cases the sweep stream keeps the water vapor on the permeate side of the membrane from condensing. This sweep is typically too high to simply vent into the environment. Consequently the permeate stream (including sweep) was compressed, dehydrated and fed back to the feed stream. In some applications, the compressor for the permeate stream is not needed and thus the capital cost of the membrane process can be reduced significantly. This includes applications where there is a use for the permeate stream as a fuel or where the feed gas is compressed from low pressure prior to dehydration. In the latter case, the permeate stream can be added to the feed flow. This only results in an incremental impact on the compressor size and cost. These scenarios were not considered in this analysis. Only the case where a separate compressor was used to recompress the permeate stream and the combined permeate and sweep streams were added back to the feed was considered in this economic evaluation.

The economics of the membrane process for natural gas dehydration was compared with that of the TEG dehydration process, which is the most wildly used technology in natural gas dehydration. The comparison was made at following operating conditions and product specifications:

1) The operating pressure was assumed to be 1000 psig as it is a very common pressure used for the pipeline gas;

2) The feed gas was assumed to be $90^{\circ} \mathrm{F}$ and saturated (at $1000 \mathrm{psig}$ );

3) Four different feed flow rates were considered in the evaluation, i.e.: 1 MMSCFD, 5 MMSCFD, 50 MMSCFD and 100 MMSCFD;

4) The natural gas pipeline specification for water vapor was $4 \mathrm{lbs} . \mathrm{H}_{2} \mathrm{O} / \mathrm{MMSCF}$ for the TEG process and $5 \mathrm{lbs}$. $\mathrm{H}_{2} \mathrm{O} / \mathrm{MMSCF}$ for the membrane process. These pipeline specifications are adopted from the industrial standard and are much more stringent than the typical 7 lbs. $\mathrm{H}_{2} \mathrm{O} / \mathrm{MMSCF}$ pipeline specification. This was due to the safety margin consideration [1]. TEG process has a higher failure rate of its mechanical parts. The membrane process is more reliable. Consequently the product gas specification for the TEG process was somewhat more stringent as compared to the membrane process specification used in this study.

The basis for the TEG process analysis was provided by the paper titled, "Cost of Conditioning Your Natural Gas for Market" [1]. The data in this paper were based on the 1992 costs. The cost data for the TEG systems were scaled from 1992 by $3 \%$ per year to make them equivalent to 2002 dollars. Operating costs 
were used as they appeared in the article. A life of four years was assumed in the article, with a declining production over that period. The same analysis method was used in the present evaluation. The need to control aromatic hydrocarbons such as benzene, toluene, ethylbenzene, and xylene (BTEX) was not considered in this article. This could add significantly to the cost of the TEG system in areas where the emission of BTEX is or will be controlled.

For the membrane process, an effort was made to optimize the operating variables. Considerable work had been done to find the optimum sweep flow rate, which had a significant impact on the capital cost and total treatment cost. The required membrane area at the optimum sweep flow rate was calculated from the computer program. This area was then divided into standard commercial size modules. The cost of each membrane module was estimated and the data used to obtain the total capital cost of the membrane skid (including the housing shell and related valves). The cost of the compressor was assumed to vary from $\$ 2000 / \mathrm{hp}$ for small units down to $\$ 700 / \mathrm{hp}$ for large units of over $1000 \mathrm{hp}$. A four year membrane life was assumed, which is the same as the life of the TEG system. The natural gas dehydration membrane should have a useful life of somewhere between the $\mathrm{CO}_{2}$ membrane service and the $\mathrm{H}_{2}$ membrane service. Membranes for $\mathrm{CO}_{2}$ removal from natural gas have demonstrated lives from 3 to 7 years. Membranes for $\mathrm{H}_{2}$ and air separation services have shown lives of 10 years and above. Consequently the life of the dehydration membrane should be much longer than the 4 year life requirement used in this study; therefore no replacement costs were included in the evaluation. There was also no credit given in the evaluation for the remainder of the membrane life. In reality either a producer or a systems lessor that owns this equipment will be able to take advantage of this longer membrane life.

As discussed above, it has been concluded that some of the hollow fibers in the module were likely blinded with tube sheet glue and therefore were not capable of passing the sweep gas or permeating water vapor. The calculations of water vapor permeances do not take this into account, but assume that the module was working properly. In order to understand how the membrane system would compare if it had worked as expected, data from a previous small-scale test with a medium pressure membrane variant were used in an equivalent economic comparison. Consequently two sets of membrane module performance properties were used in the economic analysis of the membrane process. One was from the results of this field test and the other from the results of the previous small-scale test on a medium pressure dehydration membrane conducted at 750 psig.

The economic evaluation indicated that the membrane process was competitive with the TEG process for the natural gas feed flow rate below 10 MMSCFD for the membrane with the previously measured water vapor permeance. The membrane process was competitive for the feed flow rate below 1 MMSCFD 
even for the membrane with the water vapor permeance of this field test. In all cases the simplicity of membrane operation led to savings in operating cost.

It should be noted that the use of membranes in natural gas dehydration can provide the advantage of reducing BTEX emission. Traditional TEG process does not provide a satisfied solution to the BTEX emission control. A large portion of BTEX in the feed stream is emitted to the environment unless additional processes are employed to capture the emissions. The BTEX component permeances of the dehydration membrane are at least an order of magnitude lower than the permeance of methane. Consequently only a small fraction of BTEX in the feed will permeate through the membrane. Nearly all of BTEX will remain in the residual product stream. Furthermore in the membrane process the permeate is used as a fuel or is recompressed and added back to the feed. BTEX is not emitted to the atmosphere. This increases the economic competitiveness of the membrane process. 


\section{CONCLUSION}

The field test showed that a commercial-size membrane module for natural gas dehydration was successfully fabricated. The module operated reliably at pressures exceeding 1000 psig in the field test. The effects of feed gas pressure, permeate gas pressure, feed flow rate, purge ratio, and feed gas dew point on membrane module performance were determined and found to meet the design expectations. Although the water vapor permeance was lower than the design expectation, substantial dehydration was demonstrated with low purge ratio. However the bore side pressure drop was higher than the value projected from fluid dynamic calculation. It was likely resulted from the insufficient fiber openness in either the sweep or the permeate tube sheet end. This could help explain the relatively lower water vapor permeances that were measured in the field test.

The economic evaluation of natural gas dehydration was performed for the membrane process and the traditional TEG process and the economics of these two processes were compared. Two sets of membrane module performance properties were used in the economic analysis of the membrane process. One was from the results of this field test and the other from the results of the previous small-scale test with a medium pressure dehydration membrane variant conducted at $750 \mathrm{psig}$ that exhibited higher water vapor permeance. The membrane process was competitive with the TEG process for the natural gas feed flow rate below 10 MMSCFD for the membrane with higher water vapor permeance. The membrane process was competitive for the feed flow rate below 1 MMSCFD even for the membrane with the water vapor permeance of this field test.

\section{REFERENCE}

[1] Carter Tannerhill, Linda Echterhoff, and Dennis Leppin, "Cost of Conditioning Your Natural Gas for Market", Proceedings of The Seventy Third GPA Annual Convention, 1994. 